\title{
Health Promotion Board-Ministry of Health Clinical Practice Guidelines: Falls Prevention among Older Adults Living in the Community
}

\author{
Thilagaratnam Shyamala, Sweet Fun Wong, Akila Andiappan, Kah Guan Au Eong, Anu Birla Bakshi, Debbie Boey, \\ Tsung Wei Chong, Hui Ping Eng, Noor Hafizah Ismail, Tang Ching Lau, Wei-Yen Lim, Hsin Wei Wendy Lim,
} Lydia Seong, Wei Chin Wong, Kai Zhen Yap, Sri Yudah

\begin{abstract}
The Health Promotion Board (HPB) has developed the Clinical Practice Guidelines (CPG) on Falls Prevention among Older Adults Living in the Community to provide health professionals in Singapore with recommendations for evidence-based assessments and interventions for falls prevention. This article reproduces the introduction and executive summary of the key recommendations from the HPB-MOH CPG on Falls Prevention among Older Adults Living in the Community for the information of SMJ readers. The chapters and page numbers mentioned in the reproduced extract refer to the full text of the guidelines, which are available from the Health Promotion Board website: http://www.hpb.gov. $\mathrm{sg} / \mathrm{cpg}$-falls-prevention. The recommendations should be used with reference to the full text of the guidelines. Following this article are multiple choice questions based on the full text of the guidelines.
\end{abstract}

\section{INTRODUCTION}

This Clinical Practice Guidelines on Falls Prevention among Older Adults living in the Community was developed by the Health Promotion Board to provide health professionals in Singapore with recommendations for evidence-based assessments and interventions for falls prevention.

\subsection{Objectives of guidelines}

The aim of these guidelines is to assist health professionals to identify and assess the falls risk status of older adults living in the community and to deliver evidence-based interventions for falls prevention.

\subsection{Target users}

The guidelines are intended for all health professionals, including doctors, psychologists, pharmacists, dieticians, optometrists, social workers, occupational therapists, physiotherapists and nurses to assist them in the assessment and prevention of falls in older adults.

\subsection{Epidemiology}

\subsubsection{Definition of falls}

This set of clinical practice guidelines has adopted a broader definition in order to be more sensitive in identifying falls in older adults, and has defined a fall as "a sudden, unintentional change in position causing an individual to land at a lower level (either on an object or on the floor) other than as a consequence of overwhelming external force."

\subsubsection{Incidence of falls}

According to the National Registry of Disease Office (NRDO) of Singapore, the crude incidence rate of unintentional falls in year 2012 was 277.7 per 100,000 for adults aged 60 years and older. The incidence rate increases sharply with age (Fig. 1). The majority of falls $(63 \%)$ occur at home.

$58 \%(1,448)$ of unintended falls occurred among adults aged 65 years or older according to the National Trauma Registry Report, in year 2012.

A local retrospective study conducted by Chan and colleagues (1997) found a one-year falls incidence rate of 17.2 per 100 in adults aged 60 years and above who were living in the community $(n=332)$. Of those who fell, two-thirds $(67 \%)$ had a single fall and one-third (33\%) had recurrent falls in the past one year.

In summary, falls risk is relatively high. Asian studies, including local ones, suggest that at least $20 \%$ of older adults (65 years and above) would fall in a year.

\section{Falls in Asians compared to other ethnic groups}

There is some data from Western countries that there are ethnic differences in fall injury rates, with higher rates in whites compared to people of Asian ethnicity. It is believed that the prevalence of osteoporosis (which is higher in white populations than in Asian populations) may explain some of these differences.

\subsubsection{Risk factors}

Key factors that have been identified include:

(a) Socio-demographic factors

Age is the main risk factor, with older adults at higher risk. There appear to also be ethnic and gender differences. Asians in general have a lower risk compared to other ethnic groups, while females appear to be at higher risk. Living 


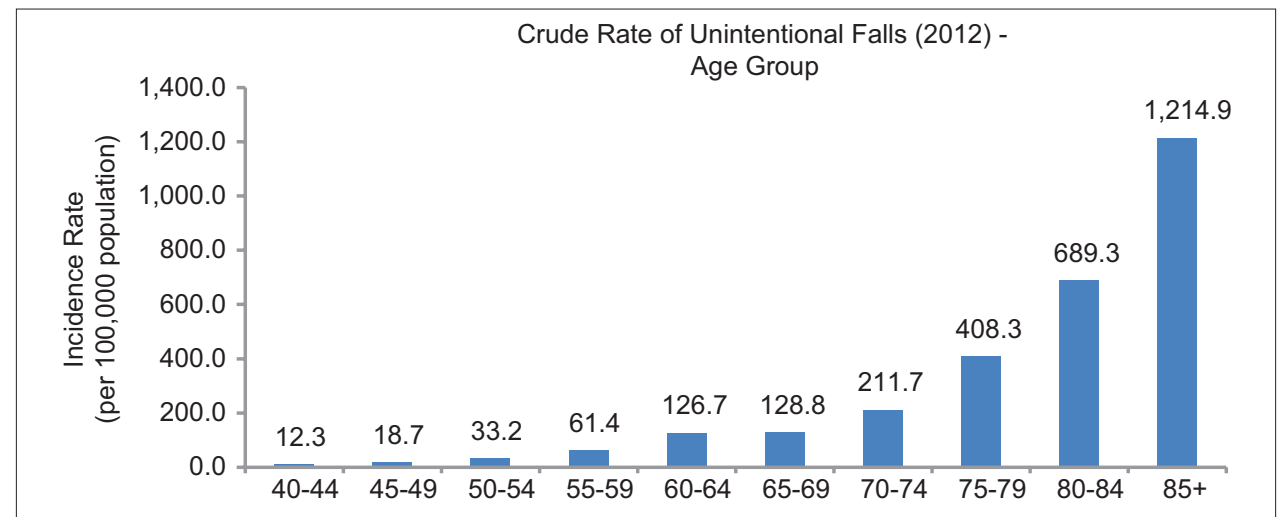

Fig. 1 Crude rate of unintentional falls [National Registry of Disease Office, Singapore. Crude rate of Unintentional falls. 2012].

alone and being single or widowed are also associated with higher falls risk.

(b) Past medical history

A past history of falls is a significant predictor of future falls risk. Neuromuscular conditions resulting in mobility impairment, gait deficit and balance deficit such as vertigo/giddiness, arthritis, Parkinson's disease and strokes can predispose older adults to falls. Sensory impairment including visual impairment and hearing loss also increases risk of falls, as do cognitive impairment and dementia. Polypharmacy and the use of certain medications have been associated with an increase in the risk of falls. Drugs that have been implicated include anti-hypertensives, hypnotics, tranquilisers/sedatives, anti-depressants and anti-arrhythmics.

(c) Extrinsic factors

The living environment also poses risks. These include use of inappropriate footwear, and environmental and home hazards. Personal factors and extrinsic factors can also interact to increase risks; and the level of risk of an environment may depend on the person. For example, an older adult with neuromuscular conditions and poor vision may have a higher risk of falls than an older adult without these intrinsic conditions living in the same housing environment. It is therefore important when assessing home and environmental hazards to consider the personal risk factors of the person who is living in the home or environment.

\section{EXECUTIVE SUMMARY OF KEY GUIDELINE RECOMMENDATIONS}

Details of recommendations can be found in the main text at the pages indicated.

\section{Falls Risk Assessment}

GPP All older adults (65 years old and older) during a healthcare encounter should be asked for history of falls and gait and balance problem (pg 10).
Older adults who have fallen more than once in 6 months or with gait and balance deficits should be offered a comprehensive fall assessment (pg 10).

Grade D, Level 3

\section{Single Interventions}

A Exercise programmes for falls prevention should consist of at least a twice-weekly programme for more than 25 weeks, with each session lasting for 60 minutes (pg 13).

Grade A, Level 1+

A Exercise intensity can be pegged at a moderate level. These exercises should be progressive and individualised to maximise the effectiveness of the programme (pg 14).

Grade A, Level 1+

A Exercises should consist of a mix of balance, lower limb strengthening (such as strengthening exercise for hip flexors, knee extensors and ankle plantar flexors), endurance and flexibility training (pg 14).

Grade A, Level 1+

B Older adults assessed to have a high risk of falls, history of falls or those with visual impairment should be referred to occupational therapists for home assessment and modification intervention (pg 15)

Grade B, Level 1+

GPP Older adults should be advised to wear well-fitting shoes with low heeled, slip resistant soles and a large contact area to reduce falls (pg 15).

GPP

B Medication review and modification to optimise medication use should be provided by the primary care physician in collaboration with a pharmacist (if available), clinical specialist (s) (in cases where specific medications are prescribed by them), the individual older adult and his/her primary caregiver where applicable, 
for older adults who are taking medications that can increase their risk for falls. This can be performed either as a single intervention or part of a multi-factorial intervention (pg 16).

Grade B, Level 1+

B Psychotropic medications (benzodiazepines and antipsychotics) should be discontinued in older adults (if possible) to prevent falls. This should be done with appropriate tapering of dose, close monitoring of outcomes and input from clinical specialists if necessary (pg 16).

Grade B, Level 1+

D It may be helpful to give vitamin D supplementation to older adults if their vitamin D level is less than $20 \mathrm{ng}$ per millilitre (50 nmol per litre), if higher levels cannot be achieved via sunlight or dietary supplementation. Older adults with significant renal impairment will benefit from treatment with analogues of vitamin D (alfacalcidol or calcitriol) (pg 17).

Grade D, Level 4

B Avoid the use of high dose cholecalciferol (500,000 IU per year) (pg 17).

Grade B, Level 1+

Older adults who have impaired vision should be referred for further evaluation of the cause of impairment (pg 18).

GPP
B Persons with cataract as the main cause of vision impairment should be referred for cataract surgery (especially for the first eye) (pg 18).

Grade B, Level 1+

B Older adults with suspected cardiogenic falls should be referred to the cardiologist for further evaluation. Cardiac pacing is recommended for patients with cardioinhibitory carotid sinus hypersensitivity and a history of falls (pg 18).

Grade B, Level 1+

GPP Provide basic information on falls and educate older adults on the benefits of falls prevention strategies in preventing falls and maintaining independence (pg 19).

GPP

\section{Multiple Interventions}

B Older adults at risk of falls should be considered for referral to falls prevention programmes consisting of exercise and other interventions, if such programmes are available and accessible (pg 19).

Grade B, Level 1+

\section{Multi-factorial Interventions}

B Older adults assessed to be at high risk of falls should receive interventions targeted at the individually identified risk factors (pg 19).

Grade B, Level 1+ 


\section{SINGAPORE MEDICAL COUNCIL CATEGORY 3B CME PROGRAMME (Code SMJ 201505B)}

These questions are based on the full text of the guidelines, which may be found at http://www.hpb.gov.sg/cpg-falls-prevention.

Question 1. Regarding incidence of falls and risk factors for falls:

(a) Male older adults are at higher risk of falls compared to female older adults.

(b) A significant predictor of future falls risk is a history of a fall in the past one year.

(c) Neuromuscular conditions resulting in mobility impairment, gait deficit and balance deficit together with polypharmacy pre-disposes older adults to falls.

(d) As the number of risk factors increase, the risk for falls increases.

Question 2. Regarding screening:

(a) All older adults should be asked for a past history of falls.

(b) General practitioners or other healthcare professionals can utilise multi-factorial assessment tools to identify older adults who are at risk of falls.

(c) If gait and balance problems are observed, a comprehensive falls assessment is recommended.

(d) Functional Mobility Assessment tools look at strength, balance, gait and reaction time.

Question 3. Regarding falls prevention interventions:

(a) Exercises consisting of two or more aspects of strength, balance, flexibility or endurance are not effective in reducing the rate of falls in older adults.

(b) Home assessment and modification interventions conducted by occupational therapists are effective in reducing the rate of falls especially for those who have a high risk of falls, history of falls or those with visual impairments.

(c) Vitamin D deficiency is uncommon in Singapore due to the sunny weather.

(d) First eye cataract surgery can reduce the risks of falls and the rate of falls in older adults.

Question 4. Regarding falls prevention interventions:

(a) Providing basic information about the benefits of falls prevention can help older adults make informed choices about engaging in falls prevention interventions and improve adherence to these interventions.

(b) Multi-factorial interventions that target specific risk factors have been shown to be effective in preventing falls.

(c) Older adults with high risk of falls should receive interventions targeted at the individually identified risk factors.

(d) Active review and modification of medications associated with increased risks of falls is recommended as a form of falls prevention intervention.

Question 5. Regarding consequences and cost of falls:

(a) Besides the physiological trauma of a fall, psychological fear of falling is real and may lead to selfimposed mobility restriction.

(b) Head injury and fractures are major causes of morbidity and mortality following a fall.

(c) The most common fracture sites from falls include Colles' fracture of the wrist, fractures of upper and lower limbs and hip fractures.

(d) Hip fractures predominate in older adults between the ages of 65 and 75.

\section{Doctor's particulars:}

Name in full

MCR number

Email address

\section{SUBMISSION INSTRUCTIONS:}

(1) Log on at the SMJ website: http://www.sma.org.sg/publications/smjcurrentissue.aspx and select the appropriate set of questions. (2) Provide your name, email address and MCR number. (3) Select your answers and click "Submit".

\section{RESULTS:}

(1) Answers will be published in the SMJ July 2015 issue. (2) The MCR numbers of successful candidates will be posted online at the SMJ website by 29 June 2015. (3) Passing mark is $60 \%$. No mark will be deducted for incorrect answers. (4) The SMJ editorial office will submit the list of successful candidates to the Singapore Medical Council. (5) One CME point is awarded for successful candidates.

Deadline for submission: (May 2015 SMJ 3B CME programme): 12 noon, 22 June 2015. 